\title{
Atomistic Modeling of Resistivity Evolution of Copper Nanoparticle in Intense Pulsed Light Sintering Process
}

\author{
Lingbin Meng, Yi Zhang, Xuehui Yang, Jing Zhang* \\ Department of Mechanical and Energy Engineering, Indiana University-Purdue University \\ Indianapolis, Indianapolis, IN 46202, USA \\ *Email: jz29@iupui.edu; Phone: 317-278-7186; Fax: 317-274-9744
}

\begin{abstract}
In this work, the intense pulsed light (IPL) sintering process of copper nanoparticle ink is simulated using molecular dynamics (MD) method. First, the neck size growth between the two copper nanoparticles during the IPL sintering process is computed. The resultant electrical resistivity is then calculated by substituting the neck size into the Reimann-Weber formula. Overall, a rapid decrease of electric resistivity is observed in the beginning of the sintering, which is caused by quick neck size growth, followed by a gradually decrease of resistivity. In addition, the correlation of the simulated temperature dependent resistivity is similar to that of the experimentally measured resistivity. The MD model is an effective tool for designers to optimize the IPL sintering process.
\end{abstract}

Keywords: copper; nanoparticle; molecular dynamics; electrical resistivity; intense pulsed light

\section{Introduction}

In recent years, sintering process of nanoparticle ink using intense pulsed light (IPL) becomes a popular technique for flexible electronics [1-7]. Rapid low-temperature sintering of nanoparticles into patterns and thin films on a large area is an attractive feature for fabrication of flexible electronics. IPL process has several advantages over the conventional sintering techniques, e.g., furnace-based, microwave, plasma, and electrical methods, including low temperature, fast sintering rate, and large area. Since the substrate of current flexible electronics is made by polymer [8], the processing temperature of conductive network on the substrate is limited. Benefiting from the low processing temperature [9], IPL becomes a strong alternative for flexible electronics manufacturing. Owing high surface area to volume ratio, nanoparticles possess 
extremely lower melting temperature than their bulk forms $[10,11]$, because surface atoms have fewer neighbors and are more weakly bound and less constrained in their thermal motion than atoms in the particles [12]. Therefore, nanoparticles are suitable nanoinks in the low processing temperature in IPL technique. In a typical IPL system, IPL is generated using an arc plasma phenomenon in the xenon flash lamp [4]. Nanoink with metal nanoparticles of about $5 \mathrm{~nm}$ diameter are uniformly dispersed on the top of polymer substrate [5]. When the lamp is triggered using a pulse controller, approximately $1000 \mathrm{~A}$ of electrical current is delivered within milliseconds from the supercapacitor $(40,000 \mu \mathrm{F})[4]$. The optical spectrum of light from the xenon lamp covers a range of wavelengths from $160 \mathrm{~nm}$ to $2.5 \mu \mathrm{m}$ [4]. Nanoink is then sintered by the IPL to form conductive network on flexible polymer substrate.

In printed electronics field, the electrical conductivity is a key property determining the performance of the printed products [13]. Regarding the material selection, though metals such as gold and silver possess high electrical conductivity, they are too expensive to achieve mass production. Copper is a popular material in flexible electronics $[2,5-7,13]$ due to its low cost and excellent electrical conductivity. Besides material selection, the electrical conductivity is mainly affected by the sintering behavior of nanoparticles [13]. Therefore, this study focuses on the electrical resistivity of the sintered copper nanoparticles.

The electrical resistivity of copper nanoparticles sintered by IPL has been studied from experimental measurements [5-7]. Kim et al. [5] tested the resistivity of sintered copper nanoink with light intensity in a range of $20-50 \mathrm{~J} / \mathrm{cm}^{2}$. In their result, the resistivity is decreased with increased pulse energy. The polymer substrate remained undamaged under a pulse energy of 50 $\mathrm{J} / \mathrm{cm}^{2}$, and the resultant resistivity is $5 \mu \Omega \cdot \mathrm{cm}$. Wang et al. [7] tested the copper nanoink resistivity under a wide range of pulse energy. They found that the pulse energy of $60 \mathrm{~J} / \mathrm{cm}^{2}$ is an inflection point of the resistivity. When the pulse energy exceeds $60 \mathrm{~J} / \mathrm{cm}^{2}$, bubbling arose on the surface of $\mathrm{Cu}$ film, so the resistivity will increase due to the increasing pores created by the bubbling. If the pulse energy is over $70 \mathrm{~J} / \mathrm{cm}^{2}$, the polymer substrate will be damaged. The resultant resistivity in their study is $3.21-5.27 \mu \Omega \cdot \mathrm{cm}$ when the light energy ranged from 40 to 60 $\mathrm{J} / \mathrm{cm}^{2}$. Overall, the experimental results show that the sintering temperature is a key factor of resultant electrical conductivity. 
To explain the temperature-dependent resistivity, it is imperative to understand the sintering process of copper nanoparticles and its influence on the performance. However, the sintering behavior during the sintering process is hard to be recorded because the sintering time is too short. Therefore, there is a need of an effective model to simulate the sintering process and predict the resultant properties. Forerunners developed many molecular dynamics (MD) models to simulate the sintering process. Zhu and Averback [14] developed a MD model to simulate the sintering process of two single-crystal copper nanoparticles at $700 \mathrm{~K}$. They found that the timescale of the whole sintering process is only tens of picoseconds [14]. Pan et al. [15] studied the sintering process of two gold nanoparticles. They developed an analytical model to compute the electrical resistivity by neck growth, and their results are consistent with the MD simulation predictions [15]. Zhang et al. [16] studied the resistivity evolutions of two silver nanoparticles at two different temperatures and found that the resistivity of sintered silver nanoparticles at higher temperature is lower due to the larger neck size. These imply that molecular dynamics model is effective to simulate the sintering process in IPL technique. In spite of the efforts of forerunners, effective simulation of electric resistivity in low temperature sintering process of copper is rarely reported. If an effective simulation model is developed, designers can make use of it to optimize the IPL sintering process.

For metal and alloy systems, the atomistic interaction potentials are typically represented by the embedded atom method (EAM) [17], which is a semi-empirical approach. EAM is effective for predicting material properties such as thermal expansion, melting temperatures, phase transformations, surface energies and vacancy formation energies, and simulation of structural behaviors such as grain boundaries and dislocations [17]. Besides the aforementioned MD simulations, $\mathrm{MD}$ is widely applied to low-dimensional systems such as nanowires [18-21], thin films [22, 23] and nanoparticles [24-26], which indicates that EAM is capable to apply to lowdimensional systems. Therefore, EAM is employed in this study.

In this paper, a molecular dynamics model of sintering of copper nanoparticles at varied temperature is developed. The neck growth between two copper nanoparticles during the 
sintering process is studied. The resistivity is calculated by the neck size using Reimann-Weber formula and then compared against experimental result.

\section{Method}

\subsection{Resistivity computation}

In sintering process, neck size is the dominant factor of electrical resistivity, because with increased neck size, the contact cross-sectional area between the two particles increases, and hence the resistivity reduces. The normalized electrical resistivity $(r)$ can be computed by Reimann-Weber formula [27]:

$$
r=\frac{\lambda_{s}}{\lambda_{e}}=\frac{1}{x}+\frac{1}{\pi} \ln \frac{2}{x}
$$

Here $\lambda_{e}$ is the effective conductivity, $\lambda_{s}$ is the conductivity for bulk copper and $x$ is the neck size $(X)$ normalized by particle radius $(D)$, such that $x=X / D$.

To extract the resultant electrical resistivity, it is necessary to obtain the neck size from the molecular dynamics model.

\subsection{Molecular dynamics model}

A molecular dynamics model is developed to simulate the sintering process at atomistic scale. The atomistic interactions are represented by the embedded atom method. In EAM, the total energy $\left(E_{t o t}\right)$ of an atomistic system is represented by the summation of energy of all atoms in the system, and the energy of atoms is calculated based on atom positions as in the following equation [28]:

$$
E_{t o t}=\frac{1}{2} \sum_{i j} V_{i j}\left(r_{i j}\right)+\sum_{i} F_{i}\left(\bar{\rho}_{i}\right)
$$

Here $V_{i j}$ is the pairwise interaction energy between atoms $i$ and $j$ separated by a distance $r_{i j}$, and $F_{i}$ is the embedded energy of atom $i$ as a function of the host electron density $\bar{\rho}_{i}$, which is also based on atom position as:

$$
\bar{\rho}_{i}=\sum_{i \neq j} \rho_{i}\left(r_{i j}\right)
$$

where $\rho(r)$ is the electron density function calculated by density functional theory (DFT) [28]. 
The sintering process of copper nanoparticles is simulated by the Large-scale Atomic/Molecular Massively Parallel Simulator (LAMMPS) [29]. The motion of all atoms in the system is calculated with a time step of 0.002 ps by integrating the atomistic equations of motion based on the potential function using velocity-Verlet numeric integrator. In each time step, the displacement of each atom is computed from the atomistic positions of previous step. The structure of a copper nanoparticle is modeled by a spherical particle cut from a large FCC single crystal supercell within a radius of $3.0 \mathrm{~nm}$. The number of atoms in the single copper nanoparticle is 9564. This nanoparticle is then duplicated to form a two-particle sintering model. The two nanoparticles are placed in a non-periodic simulation box with shrink-wrapped boundaries to form an isolated system [16], with an initial center-to-center distance of $6.2 \mathrm{~nm}$, as shown in Fig. 1. The whole system is subject to constant temperatures by rescaling the atom velocity with NVT ensemble [16] for 80 ps. The constant temperatures are maintained by NoseHoover thermostat. This timescale is sufficient for the neck size of the two nanoparticles being stable [14]. To avoid translation and rotation of the system, the centers of mass and angular momentum for the whole system are eliminated. During the simulation, the position of center of mass for each nanoparticle as well as the positions and velocities of all atoms are recorded at every $0.1 \mathrm{ps}$. The sintering processes of copper nanoparticles at temperatures of $150^{\circ} \mathrm{C}, 200^{\circ} \mathrm{C}$, $250^{\circ} \mathrm{C}, 300^{\circ} \mathrm{C}$ and $350^{\circ} \mathrm{C}$ are simulated. Additional computational details refer to Ref. [16].

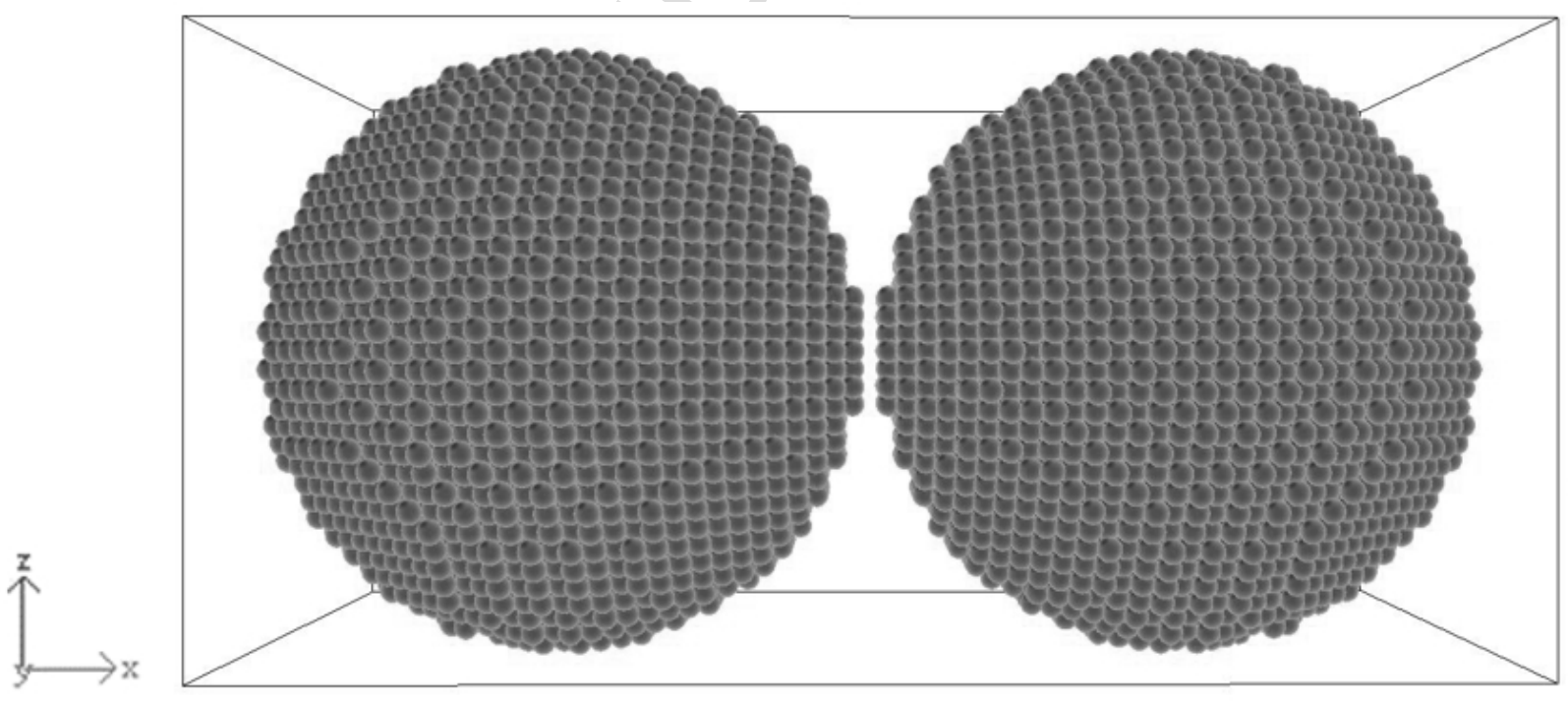

Fig. 1. System of two copper nanoparticles 
Once the computation of simulation is completed, the configuration changes of the two nanoparticles can then be extracted from the atom trajectory. Diameter of each particle is computed by the average of the upper and lower bounds of the particle. The center-to-center distance between the two nanoparticles is obtained by the positions of center of mass of the two nanoparticles. To obtain the neck size from MD computation, a dynamically allocated block region with a $0.2 \mathrm{~nm}$ thickness is defined between two nanoparticles [16]. Neck size is calculated from atoms upper and lower bounds in this region. The neck size computed by this method is consistent with the analytical results based on lattice diffusion [16].

\section{Results and discussion}

\subsection{Neck Growth}

MD simulations of the two-particle sintering model are performed in isothermal conditions of $150^{\circ} \mathrm{C}, 200^{\circ} \mathrm{C}, 250^{\circ} \mathrm{C}, 300^{\circ} \mathrm{C}$ and $350^{\circ} \mathrm{C}$. Configuration evolution of the two-particle system at $200^{\circ} \mathrm{C}$ is shown in Fig. 2. The local crystal structures are mapped on each atom according to its coordination number. The two particles are cut from their center plane to display interior atoms. The particle surfaces are amorphous whereas the interiors remain FCC crystal structure of bulk copper. The initial state of the two particles are shown in Fig. 2(a), in which the two particles are separated by initial distance of $0.2 \mathrm{~nm}$. Then, the two particles move towards each other. At $3 \mathrm{ps,}$ the initial neck formed and the gap between two particles is filled up, as shown in Fig. 2(b). At $11 \mathrm{ps}$, the neck grows rapidly and some dislocations are generated due to the formation of a plane of staking faults between the particles, as shown in Fig. 2(c). The configuration of the two particles are similar in Fig. 2(d) and (e), indicating that the neck growth becomes slower and the dislocations growth has stopped. The configuration evolution is consistent with previous MD model [16]. 


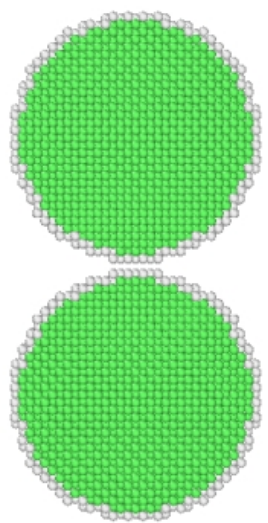

(a)

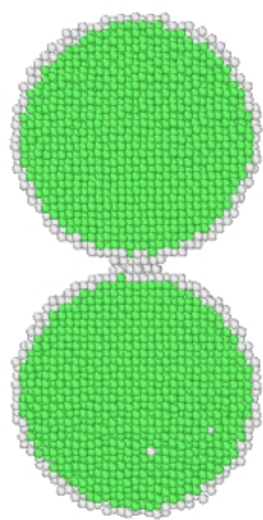

(b)

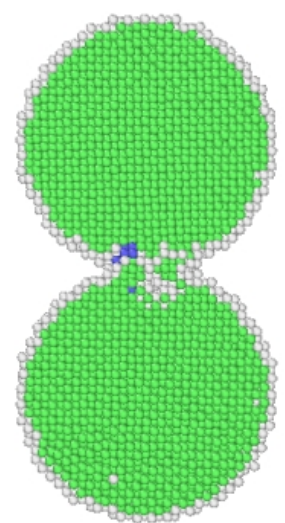

(c)

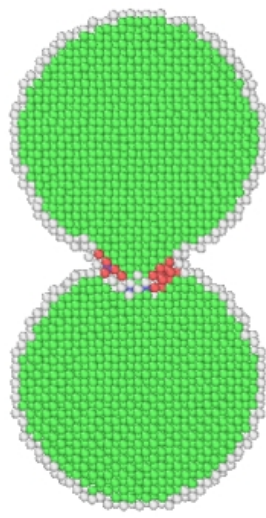

(d)

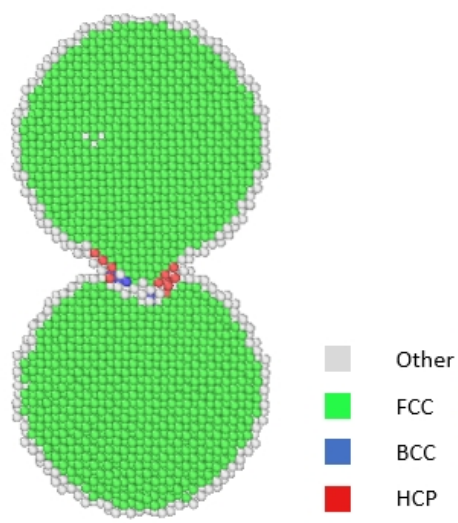

(e)

Fig. 2. Configuration evolution of the two-particle system at $200^{\circ} \mathrm{C}$ : (a) 0 ps, (b) 3 ps, (c) 11 ps, (d) $51 \mathrm{ps}$, and (e) $80 \mathrm{ps}$.

The neck growth during the sintering process at different temperatures is plotted in Fig. 3. A rapid neck growth region can be observed in the time range of 4 ps to 8 ps. The rapid sintering occurs by a dislocation mechanism due to the high shear stresses that are developed in small particle contacts which exceed the theoretical strength of the particles [14]. After the rapid neck growth region, the sintering can be considered as intermediate stage as neck growth becomes slower. Regarding temperature, the neck sizes at $250^{\circ} \mathrm{C}$ and $300^{\circ} \mathrm{C}$ are similar and larger than the neck sizes at other temperatures. Based on the trend, the final neck size will reach a maximum value at a temperature between $250^{\circ} \mathrm{C}$ and $300^{\circ} \mathrm{C}$. 


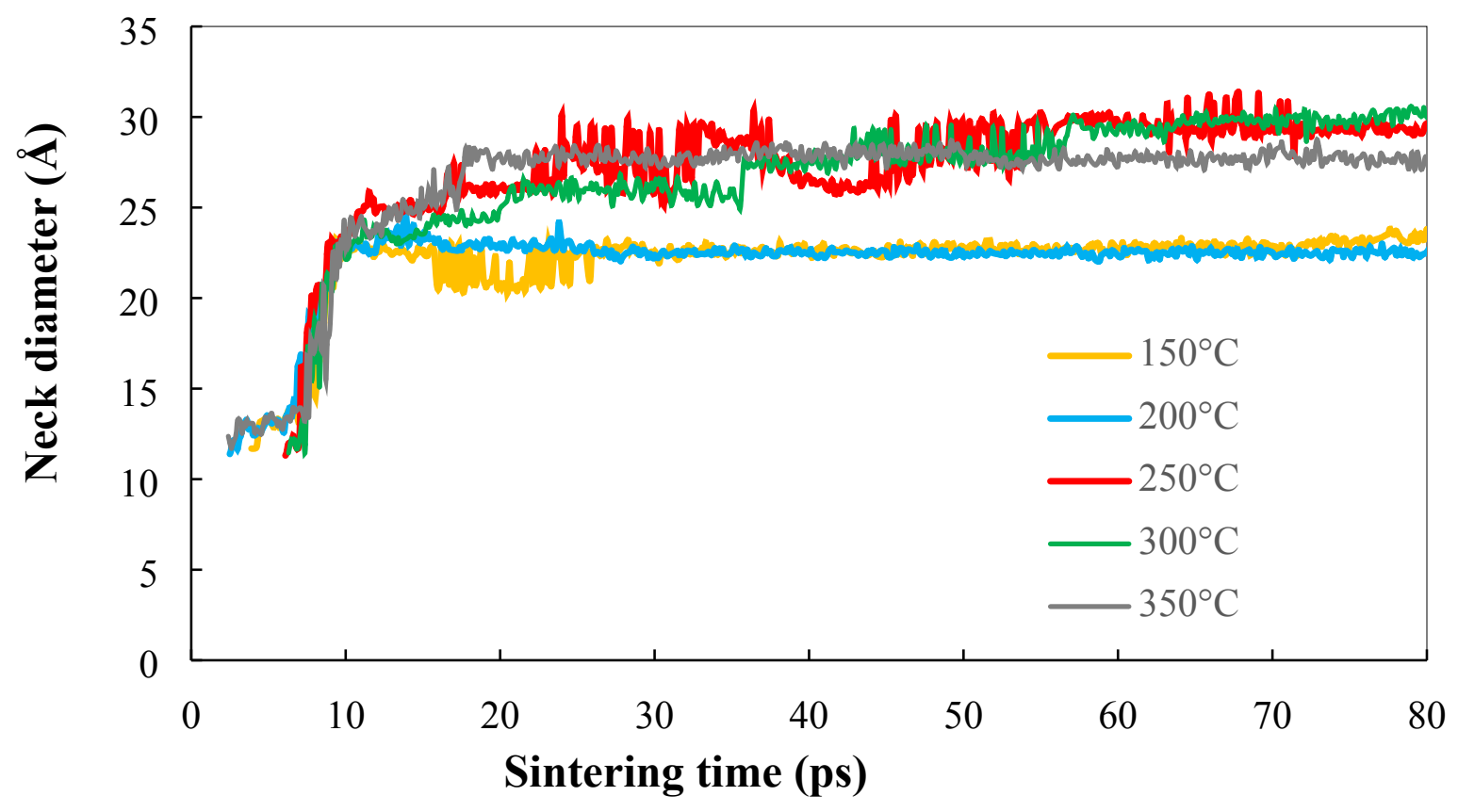

Fig. 3. Simulated neck growth with respect to sintering time at varied temperatures

\subsection{Resistivity evolution}

By substituting the neck size into Reimann-Weber formula, the normalized resistivity evolution during the sintering process can be obtained, as shown in Fig. 4. The resistivity at the initial stage has a rapid dropping region due to the rapid neck growth. As the sintering process enters the intermediate stage, the change rate of resistivity becomes low, as the neck size tends to be stable. The normalized resistivity at $250^{\circ} \mathrm{C}$ and $300^{\circ} \mathrm{C}$ are smaller than the other three temperatures, which follows the similar trends as the neck size. 


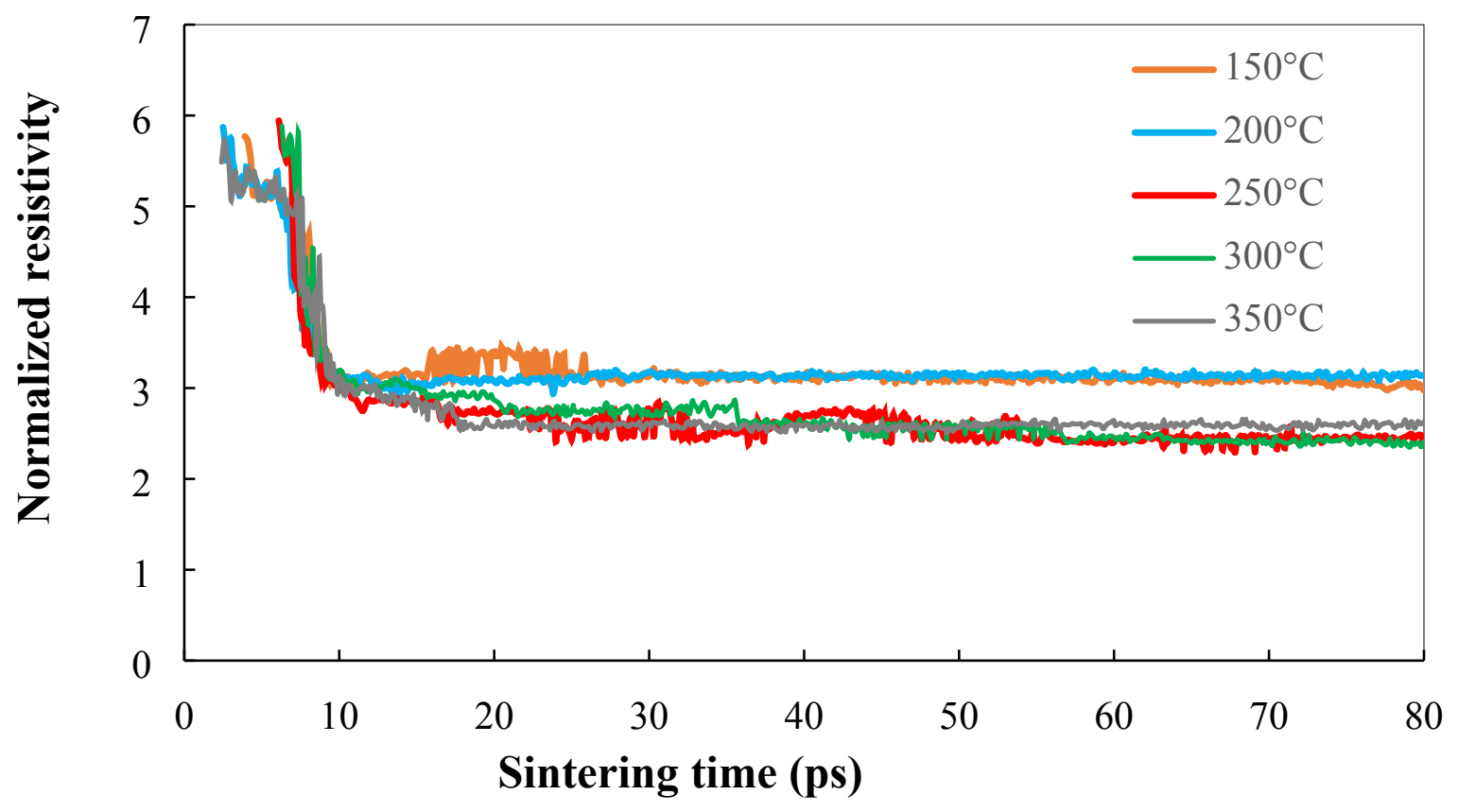

Fig. 4. Simulated normalized resistivity with respect to time at varied temperatures

To compare the simulated results to the experimental results, the resistivity from experiment [7] is normalized by the resistivity of bulk copper $(1.678 \mu \Omega \cdot \mathrm{cm})$ [30], as shown in Fig. 5. It can be observed that the normalized resistivity follows similar trend with respect to both temperatures in simulations and light intensity in experiments. The largely increased resistivity of $70 \mathrm{~J} / \mathrm{cm}^{2}$ light intensity is due to the formation of pores caused by the bubbling arose in high light intensity [7], which cannot be modeled by two-particle system. 


\section{Light intensity $(\mathrm{J} / \mathrm{cm} 2)$}

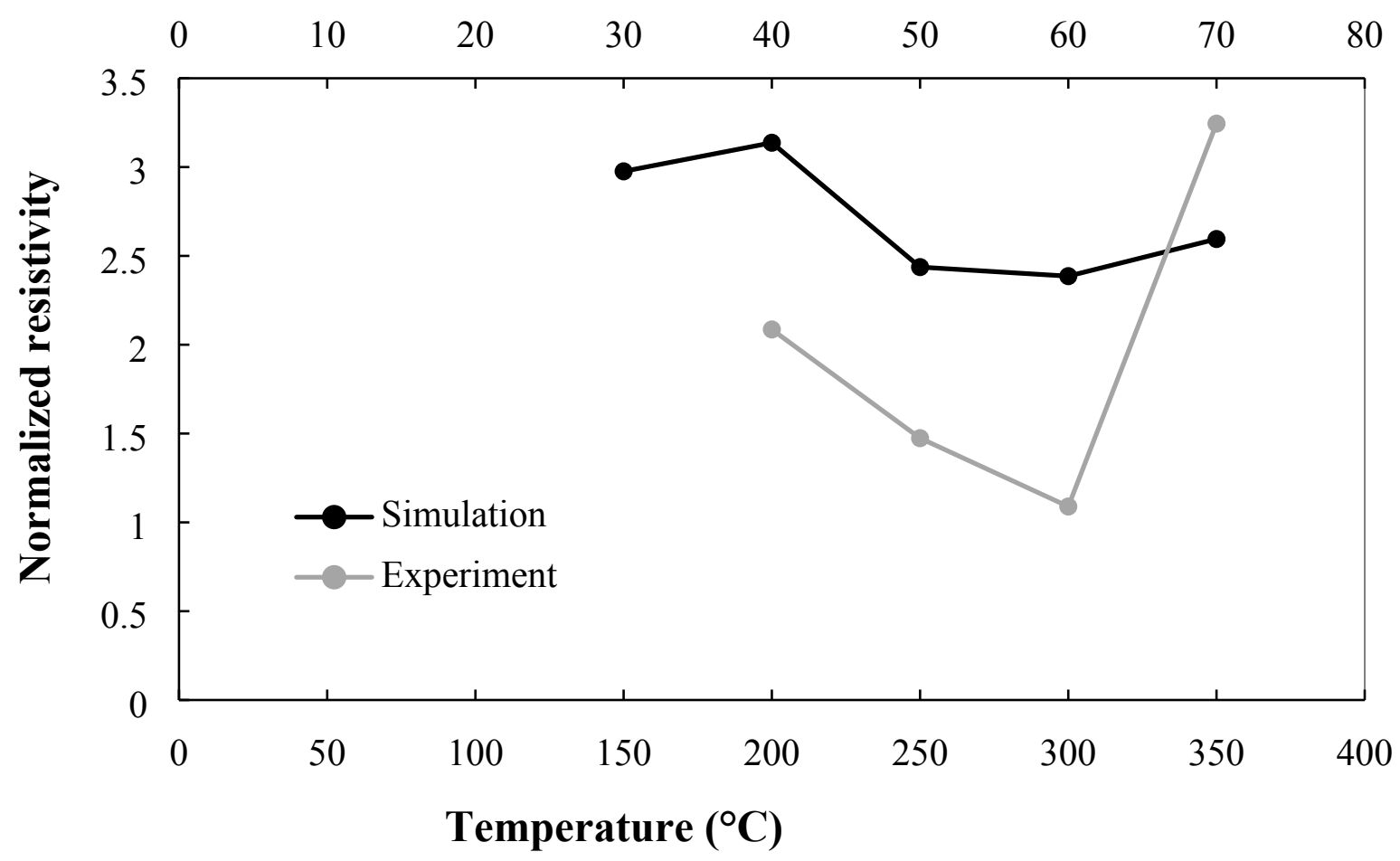

Fig. 5. Normalized resistivity with respect to temperature and light intensity, with upper abscissa being the light intensity in experiment, and lower abscissa being the temperatures in simulation

\section{Conclusions}

In this study, the sintering process of copper nanoparticles at low temperatures is simulated by MD models. The major findings are summarized as follows:

1. Neck growth during the sintering process is extracted from the MD simulations. At initial stage, the neck size increases rapidly in several picoseconds. After that, the sintering process enters an intermediate stage and change of neck size becomes much smaller.

2. Resistivity evolutions during the sintering process are computed by substituting the neck size from MD simulations into the Reimann-Weber formula. The resistivity decreases rapidly in the initial stage of sintering, due to the rapid neck growth at this stage

3. The correlation of the simulated resistivity with respect to temperature is similar to that of the experimentally measured resistivity Overall, MD models are effective tool for designers to simulate the sintering process of IPL technique. 


\section{Acknowledgment}

JZ acknowledges the financial support provided by Walmart Foundation (project title: Optimal Plastic Injection Molding Tooling Design and Production through Advanced Additive Manufacturing), and Praxair's TruForm ${ }^{\mathrm{TM}}$ AMbition Grant awarded to Indiana UniversityPurdue University Indianapolis. 


\section{References}

[1] W. H. Chung, H. J. Hwang, S. H. Lee, and H. S. Kim, "In situ monitoring of a flash light sintering process using silver nano-ink for producing flexible electronics," Nanotechnology, vol. 24, p. 035202, Jan 252013.

[2] R. Dharmadasa, M. Jha, D. A. Amos, and T. Druffel, "Room temperature synthesis of a copper ink for the intense pulsed light sintering of conductive copper films," ACS Appl Mater Interfaces, vol. 5, pp. 13227-34, Dec 262013.

[3] J. Jiu, M. Nogi, T. Sugahara, T. Tokuno, T. Araki, N. Komoda, et al., "Strongly adhesive and flexible transparent silver nanowire conductive films fabricated with a high-intensity pulsed light technique," Journal of Materials Chemistry, vol. 22, p. $23561,2012$.

[4] J. S. Kang, J. Ryu, H. S. Kim, and H. T. Hahn, "Sintering of Inkjet-Printed Silver Nanoparticles at Room Temperature Using Intense Pulsed Light," Journal of Electronic Materials, vol. 40, pp. 2268-2277, 2011.

[5] H.-S. Kim, S. R. Dhage, D.-E. Shim, and H. T. Hahn, "Intense pulsed light sintering of copper nanoink for printed electronics," Applied Physics A, vol. 97, pp. 791-798, 2009.

[6] J. Ryu, H.-S. Kim, and H. T. Hahn, "Reactive Sintering of Copper Nanoparticles Using Intense Pulsed Light for Printed Electronics," Journal of Electronic Materials, vol. 40, pp. 42-50, 2010.

[7] B. Y. Wang, T. H. Yoo, Y. W. Song, D. S. Lim, and Y. J. Oh, "Cu ion ink for a flexible substrate and highly conductive patterning by intensive pulsed light sintering," ACS Appl Mater Interfaces, vol. 5, pp. 4113-9, May 222013.

[8] M.-C. Choi, Y. Kim, and C.-S. Ha, "Polymers for flexible displays: From material selection to device applications," Progress in Polymer Science, vol. 33, pp. 581-630, 2008.

[9] J. Chung, S. Ko, C. P. Grigoropoulos, N. R. Bieri, C. Dockendorf, and D. Poulikakos, "Damage-Free Low Temperature Pulsed Laser Printing of Gold Nanoinks On Polymers," Journal of Heat Transfer, vol. 127, pp. 724-732, 2005.

[10] G. L. Allen, R. A. Bayles, W. W. Gile, and W. A. Jesser, "Small Particle Melting of Pure Metals," Thin Solid Films, vol. 144, pp. 297-308, 1986.

[11] P. Buffat and J. P. Borel, "Size effect on the melting temperature of gold particles," Physical Review A, vol. 13, pp. 2287-2298, 1976.

[12] M. Schmidt, R. Kusche, B. v. Issendorff, and H. Haberland, "Irregular variations in the melting point of size-selected atomic clusters," Nature, vol. 393, pp. 238-240, 1998.

[13] J. S. Kang, H. S. Kim, J. Ryu, H. Thomas Hahn, S. Jang, and J. W. Joung, "Inkjet printed electronics using copper nanoparticle ink," Journal of Materials Science: Materials in Electronics, vol. 21, pp. 1213-1220, 2010.

[14] H. Zhu, "Sintering processes of two nanoparticles: a study by molecular dynamics simulations," Philosophical Magazine Letters, vol. 73, pp. 27-33, 1996.

[15] H. Pan, S. H. Ko, and C. P. Grigoropoulos, "The Solid-State Neck Growth Mechanisms in Low Energy Laser Sintering of Gold Nanoparticles: A Molecular Dynamics Simulation Study," Journal of Heat Transfer, vol. 130, pp. 092404-092404, 2008.

[16] Y. Zhang, L. Wu, X. Guo, Y.-G. Jung, and J. Zhang, "Molecular dynamics simulation of electrical resistivity in sintering process of nanoparticle silver inks," Computational Materials Science, vol. 125, pp. 105-109, 2016/12/01/ 2016.

[17] M. S. Daw, S. M. Foiles, and M. I. Baskes, "The embedded-atom method: a review of theory and applications " Materials Science Reports, vol. 9, pp. 251-310, 1993. 
[18] J. Diao, K. Gall, and M. L. Dunn, "Surface-stress-induced phase transformation in metal nanowires," Nat Mater, vol. 2, pp. 656-60, Oct 2003.

[19] S. J. A. Koh, H. P. Lee, C. Lu, and Q. H. Cheng, "Molecular dynamics simulation of a solid platinum nanowire under uniaxial tensile strain: Temperature and strain-rate effects," Physical Review B, vol. 72, 2005.

[20] H. Liang, M. Upmanyu, and H. Huang, "Size-dependent elasticity of nanowires: Nonlinear effects," Physical Review B, vol. 71, 2005.

[21] H. A. Wu, "Molecular dynamics study of the mechanics of metal nanowires at finite temperature," European Journal of Mechanics - A/Solids, vol. 25, pp. 370-377, 2006.

[22] T. Fu, X. Peng, C. Huang, D. Yin, Q. Li, and Z. Wang, "Molecular dynamics simulation of VN thin films under indentation," Applied Surface Science, vol. 357, pp. 643-650, 2015.

[23] K. Zhou, T. Zhang, B. Liu, and Y. Yao, "Molecular dynamics simulations of tensile deformation of gradient nano-grained copper film," Computational Materials Science, vol. 142, pp. 389-394, 2018.

[24] L. Ding, R. L. Davidchack, and J. Pan, "A molecular dynamics study of sintering between nanoparticles," Computational Materials Science, vol. 45, pp. 247-256, 4// 2009.

[25] P. Song and D. Wen, "Molecular dynamics simulation of the sintering of metallic nanoparticles," Journal of Nanoparticle Research, vol. 12, pp. 823-829, 2009.

[26] J. Xu, R. Sakanoi, Y. Higuchi, N. Ozawa, K. Sato, T. Hashida, et al., "Molecular Dynamics Simulation of Ni Nanoparticles Sintering Process in Ni/YSZ MultiNanoparticle System," The Journal of Physical Chemistry C, vol. 117, pp. 9663-9672, 2013/05/16 2013.

[27] A. V. Luikov, Heat and Mass Transfer, handbook. Mocsow: Energia, 1971.

[28] P. L. Williams, Y. Mishin, and J. C. Hamilton, "An embedded-atom potential for the $\mathrm{Cu}-$ Ag system," Modelling and Simulation in Materials Science and Engineering, vol. 14, pp. 817-833, 2006.

[29] S. Plimpton, "Fast Parallel Algorithms for Short-Range Molecular Dynamics," Journal of Computational Physics, vol. 117, pp. 1-19, 3/1/ 1995.

[30] R. A. Matula, "Electrical resistivity of copper, gold, palladium, and silver," Journal of Physical and Chemical Reference Data, vol. 8, pp. 1147-1298, 1979. 